\title{
QUALIDADE DE BULBOS DE CEBOLA EM CONSEQUÊNCIA DE TRATAMENTOS PRÉ-COLHEITA
}

\author{
Marcos David Ferreira $^{1 *}$; Keigo Minami² \\ ${ }^{1}$ SVS do Brasil Sementes Ltda. Estação Experimental de Hortaliças, C.P. 102 - CEP: 13140-000 - Paulínia, SP. \\ 2 Depto. de Produção Vegetal - USP/ESALQ, C.P. 9 - CEP: 13418-900 - Piracicaba, SP. \\ *Autor correspondente <marcos.david.ferreira@svseeds.com.br>
}

\begin{abstract}
RESUMO: Com o objetivo de se avaliar o efeito de tratamentos pré-colheita na qualidade de bulbos de cebola, através dos parâmetros perda de peso, espessura e coloração da casca, diâmetro e firmeza do bulbo, foi conduzido experimento em Paulínia, Estado de São Paulo, em 1997. Foram utilizados quatro tratamentos:

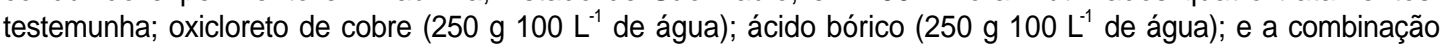
dos dois últimos e seis cultivares: Serrana, Régia, Crioula e três híbridos da SVS, HT, HS-1 e HS-2. Os tratamentos foram aplicados durante a bulbificação. Os bulbos foram armazenados à temperatura ambiente $\left(25^{\circ} \mathrm{C}\right)$ e analisados periodicamente para os parâmetros citados. Os resultados permitiram observar que a aplicação pré-colheita do oxicloreto de cobre, nas quatro últimas semanas do ciclo, aumentou significativamente a resistência da casca dos bulbos, reduziu a perda de peso e incrementou a coloração dos mesmos, para todas as cultivares. Cultivares com casca mais escura apresentaram melhor resposta aos tratamentos précolheita para a coloração, do que as de casca mais clara. A firmeza dos bulbos, não foi influenciada pelos tratamentos pré-colheita.
\end{abstract}

Palavras-chave: cebola, cor da casca, resistência da casca, micronutrientes

\section{ONION BULB QUALITY DUE TO PRE-HARVEST TREATMENTS}

\begin{abstract}
Pre-harvest treatments were evaluated to enhance onion bulb quality in different onion cultivars adapted to tropical conditions. Parameters used were weight loss, skin color and thickness, bulb and firmness.

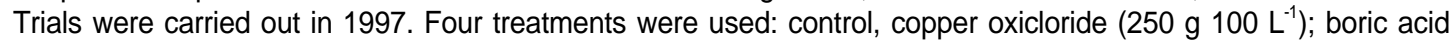
(250 g $\left.100 \mathrm{~L}^{-1}\right)$, and a combination of the two chemical treatments (250 g $100 \mathrm{~L}^{-1}$ of each). Treatments were applied at bulbing stage. The six cultivars were Serrana, Régia, Crioula, and the hybrids HT, HS-1 and HS-2. Bulbs were stored at room temperature prior to evaluations. Results showed that copper oxicloride $(250 \mathrm{~g}$ $100 \mathrm{~L}^{-1}$ ) induced an increase in skin thickness, decreased weight loss and enhanced color in cultivars. Dark skin cultivars had a better response to pre-harvest treatments in relation to light skin cultivars. Firmness was not affected by pre-harvest treatments.
\end{abstract}

Key words: onion, skin color, skin thickness, micronutrients

\section{INTRODUÇÃO}

O Brasil produziu no ano de 1998, 874.716 toneladas de cebola, classificando-se como décimo produtor mundial dessa cultura (FNP Consultoria e Comércio, 1999). Com a criação do MERCOSUL, o Brasil passou a importar da Argentina uma cebola de boa qualidade, uniforme, de sabor pungente e com a presença de casca marrom-escura. O consumidor brasileiro, diante da cebola argentina, passou a preferir esta em detrimento da nacional. Para tornarem-se competitivos no mercado, produtores brasileiros buscam por uma cebola com casca de coloração mais escura e cujo cultivo seja adaptado as condições tropicais. Na Argentina, $80 \%$ da cebola cultivada é da variedade Sintética Valenciana 14, de baixa cerosidade, ciclo longo e com casca de coloração marromescura (Acosta et al., 1993). Os campos de produção de cebola na Argentina mostram uma eficiência 50\% superior aos ganhos apresentados pelas lavouras brasileiras (Boeing, 1995). A média nacional de produtividade em cebola no Brasil está em torno de 12ton./ha, mas algumas regiões no Estado de São Paulo possuem médias de produtividade superiores (Boeing, 1995). O custo médio de produção tem variado de US\$ 0,10 a 0,15 (dez a quinze centavos de dólar) por quilo produzido (FNP Consultoria e Comércio, 1999). Há diversos fatores que afetam a qualidade e a coloração dos bulbos de cebola, como: ponto de maturação, épocas diferentes de colheita (Tucker \& Drew, 1982), e certas mudanças na nutrição (Bender, 1993; Brewster \& Butler, 1989). Knott (1933), trabalhando com produtores de cebola no estado de Nova York, Estados Unidos, relata a importância das aplicações de sulfato de cobre na coloração e qualidade dos bulbos de cebola. A aplicação de cobre intensificou a coloração da casca e os bulbos demonstraram um melhor armazenamento. Comunicação pessoal de produtores e técnicos da extensão rural, principalmente da região de Piedade, estado de São Paulo; e Ituporanga, estado de Santa Catarina, corroboram com essa hipótese. Outros micronutrientes também estão envolvidos com a qualidade dos bulbos. Calbo et al. (1986) descreve que deficiência de B ocasiona a má formação das cascas externas. Grande parte dos estudos para melhoria da qualidade dos bulbos, coloração e espessura da casca de cebola, tem 
sido realizados com cultivares de clima temperado, de ciclo longo, e sabidamente de cascas de coloração escura (Ellerbrock, 1997; Knott, 1933). A aplicação de micronutrientes nessas situações tem sido realizada via solo visando uniformizar e incrementar a coloração e a espessura da casca. Qüercitina é um dos principais compostos fenólicos presentes em catáfilos de cebola (Crozier et al., 1997; Fossen et al., 1996; Leighton et al., 1992 ). Perkin \& Hummel (1896), citados por Fenwick \& Hanley (1985), relatam a presença de qüercitina na casca de cebola tipo amarela, e também relataram o uso desta casca como tinta para pintura em fábricas de móveis no início do século. $O$ trabalho em pauta buscou avaliar o efeito da aplicação dos micronutrientes Cobre e Boro na fase pré-colheita, durante o processo de bulbificação da cebola, na formação da casca, conservação e coloração dos bulbos de cebola de diferentes cultivares adaptadas as condições tropicais.

\section{MATERIAL E MÉTODOS}

Ensaios de campo foram instalados na estação experimental de hortaliças em Paulínia (SP) no ano de 1997 , localizada na latitude $22^{\circ} 54^{\prime} S$ e longitude $47^{\circ} 05^{\prime} \mathrm{W}$, e altitude de 660 metros acima do nível do mar. O solo dessa propriedade é classificado como Latossolo Vermelho Amarelo-LVA, fase arenosa. A amostra do solo para análise foi feita anteriormente ao plantio, seguindo-se as recomendações descritas por Raij et al. (1996). A análise de solo foi realizada pelo Laboratório de Análises de Solos do Departamento de Solos e Nutrição de Plantas, da Escola Superior de Agricultura "Luiz de Queiroz", ESALQ, da Universidade de São Paulo. Foram utilizadas três variedades e três híbridos, provenientes do programa de cebolas tropicais da empresa SVS do Brasil Sementes Ltda. O sistema de cultivo e os tratos culturais utilizados foram os tradicionalmente observados pelos produtores de cebola.

Produção das mudas: Para a produção das mudas de cebola, o terreno foi previamente arado e gradeado e os canteiros construídos com 1,0 m de largura por $15 \mathrm{~cm}$ de altura, com enxada rotativa, no sentido transversal ao declive. Os canteiros possuíam $25 \mathrm{~m}$ de comprimento e corredores com $50 \mathrm{~cm}$ de largura (Ferreira, 2000). Neste experimento, não se realizou tratamento sanitário anterior à semeadura. Em cada canteiro, foram riscadas 8 linhas no sentido longitudinal. A semeadura foi manual, realizada em 5 de maio, e a densidade utilizada foi a de $3 \mathrm{~g}$ de semente $\mathrm{m}^{-2}$ de canteiro. Após a semeadura, as sementes foram cobertas, utilizando-se bagacilho de cana devidamente curtido. Semeou-se $10 \mathrm{~g}$ de cada cultivar e as cultivares foram separadas uma das outras, nos canteiros, pela distância de $20 \mathrm{~cm}$. Utilizou-se como fonte de adubo orgânico, esterco de galinha curtido, na dose de $1 \mathrm{~kg} \mathrm{~m}^{-2}$. A adubação mineral foi de $100 \mathrm{~g}$ de fertilizante, 4-14-8, por $\mathrm{m}^{2}$ de canteiro. A adubação de cobertura foi realizada 30 dias após a semeadura, utilizando-se sulfato de amônio na dosagem de $15 \mathrm{~g}$ por $\mathrm{m}^{2}$. A capina sempre foi manual, a irrigação por aspersão e as pulverizações com inseticidas e fungicidas iniciaram-se logo após a emergência, utilizando-se na fase de produção das mudas as dosagens praticadas pelos produtores e as recomendadas pelos fabricantes. Utilizaram-se os fungicidas

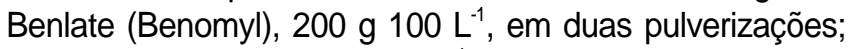

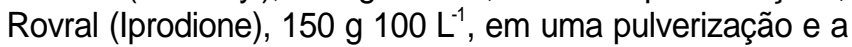

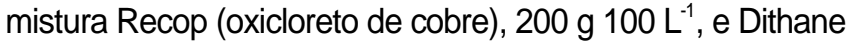
PM (Mancozeb), $200 \mathrm{~g} 100 \mathrm{~L}^{-1}$, também em uma pulverização. Foram usados diversos inseticidas para controle de insetos, seguindo-se as recomendações dos fabricantes. No canteiro de mudas ainda foram realizadas três

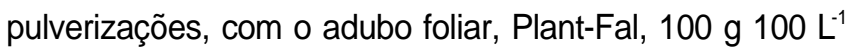
que apresenta a seguinte formulação, $\mathrm{K} 10 \%, \mathrm{Mg} 1 \%, \mathrm{Zn} 8 \%$, B 3\%, Fe $2 \%$, Mn 2\%, Cu 0,5\% e Mo 0,2\%.

Produção no campo: O terreno para transplante foi previamente arado e gradeado e os canteiros levantados utilizando-se enxada rotativa. A análise do solo (TABELA 1) permitiu estabelecer a fertilidade do mesmo, assim como planejar as adubações; os valores apresentados foram interpretados segundo Raij et al. (1996), permitindo observar os altos teores de micro e macronutrientes neste solo.

A adubação mineral e orgânica realizada após a construção dos canteiros, empregou esterco de galinha curtido, $2.000 \mathrm{~kg} \mathrm{ha}^{-1}$ e o fertilizante 4-14-8, na quantidade de $1.000 \mathrm{~kg} \mathrm{ha}^{-1}$. Após esta aplicação, realizou-se a incorporação com a enxada rotativa.

O transplante ocorreu 60 dias após a semeadura. As mudas antes de serem transplantadas foram previamente selecionadas e as ramas cortadas, com cerca de $20 \mathrm{~cm}$ de comprimento. O espaçamento utilizado para

TABELA 1 - Análise química do solo $(0-20 \mathrm{~cm}$ de profundidade) anterior ao plantio, do local do experimento, na Estação Experimental de Hortaliças SVS.

\begin{tabular}{|c|c|c|c|c|c|c|c|c|c|c|c|c|c|c|c|c|c|c|}
\hline $\mathrm{pH}$ & M.O. & $P$ & S-SO4 & $\mathrm{K}$ & $\mathrm{Ca}$ & $\mathrm{Mg}$ & $\mathrm{Al}$ & $\mathrm{H}+\mathrm{Al}$ & SB & $\mathrm{T}$ & V & $\mathrm{m}$ & B & $\mathrm{Cu}$ & $\mathrm{Fe}$ & $\mathrm{Mn}$ & $\mathrm{Zn}$ & $\mathrm{Na}$ \\
\hline $\begin{array}{c}\mathrm{CaCl} \\
0,01 \\
\mathrm{~mol} \mathrm{~L}^{-1}\end{array}$ & $\mathrm{gdm} \mathrm{d}^{-3}$ & $\mathrm{mg}$ & $\mathrm{dm}^{-3}$ & ------. & ----- & $--m$ & $\mathrm{nol}_{\mathrm{c}} \mathrm{C}$ & $m^{-3}--\cdot$ & ------- & ------- & & & ---- & ----- & $\mathrm{mg} \mathrm{c}$ & $\mathrm{Im}^{3}-$ & $\cdots$ & ---- \\
\hline 5,4 & 21 & 286 & 60,12 & 6,2 & 47 & 9 & 0 & 31 & 62,2 & 93,2 & 67 & 0 & 0,75 & 4,9 & 67 & 6,6 & 4,1 & 23,0 \\
\hline
\end{tabular}


plantio foi de $25 \mathrm{~cm}$ entre linhas e de $8 \mathrm{~cm}$ entre plantas. Foram utilizadas 4 repetições, constando de 50 plantas cada, as quais foram separadas no campo por um espaço de 20 $\mathrm{cm}$. A adubação de cobertura ocorreu aos 50 dias após o transplante utilizando-se sulfato de amônio na dosagem de $300 \mathrm{~kg} \mathrm{ha}^{-1}$. As plantas, durante o cultivo, receberam pulverizações com os fungicidas Dithane PM

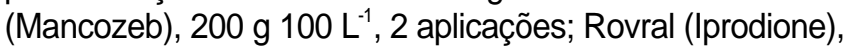

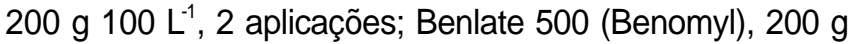
$100 \mathrm{~L}^{-1}, 2$ aplicações. O fungicida Recop (Oxicloreto de

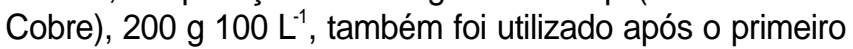
mês em 2 pulverizações. O cultivo em 1997, foi caracterizado por uma grande incidência de Tripes (Thrips tabaci), que exigiu aplicações semanais e alternadas de diversos inseticidas. O controle das plantas daninhas foi feito aplicando-se, duas semanas após o transplante, o herbicida Afalon SC (Linuron), 1,5 L ha ${ }^{-1}$, para controle em préemergência de plantas daninhas de folha larga e para controle de folhas estreitas, em pós-emergência o herbicida Fusilade 125 (Fluazifop-p-butil), 1,5 L ha-1. As dosagens utilizadas foram sempre as praticadas pelos produtores e recomendadas pelos fabricantes. A irrigação foi por aspersão, em um total de 2 horas de irrigação por semana, divididos em 3 vezes na semana, utilizando-se o bico aspersor, AgroPolo, especificação $6 \times 5$, distanciado de $18 \mathrm{~m}$ cada um. O ponto de colheita utilizado foi o tradicionalmente usado por produtores, ou seja, quando $50-60 \%$ das plantas estavam estaladas. Por ter sido uma primavera com altas temperaturas, o estalo e a maturação dos bulbos de todos os materiais foram coincidentes.

Materiais genéticos: As cultivares selecionadas para este experimento apresentam origem e coloração de casca diferenciadas. Utilizou-se as variedades Serrana, Régia e Crioula. A variedade Serrana, com casca de coloração clara; a variedade Régia, com casca mais escura que a anterior e a variedade Crioula, com presença de maior número de escamas e de coloração marrom-escura. Utilizaram-se também dois híbridos simples, HS-1 e HS-2, e um híbrido triplo HT. Os híbridos simples são provenientes do cruzamento de distintas linhas macho-estéreis, tipo Baia Periforme, com presença de cascas claras, utilizando como polinizador a variedade Crioula. O híbrido triplo é proveniente do cruzamento de um híbrido $F_{1}$, utilizando-se como polinizador a variedade Crioula. $O$ híbrido $F_{1}$, provém de um cruzamento entre duas linhagens, de populações tipo Baia Periforme.

Tratamentos: Foram utilizados quatro tratamentos: (a) Tratamento 1: testemunha. (b) Tratamento 2: oxicloreto de cobre na dosagem de $250 \mathrm{~g} 100 \mathrm{~L}^{-1}$ de água; (c) Tratamento 3: ácido bórico na dosagem de $250 \mathrm{~g} 100 \mathrm{~L}^{-1}$ de água; (d) Tratamento 4: combinação dos tratamentos 2 e 3. As aplicações foram feitas em diferentes épocas: início da bulbificação (uma aplicação), meio da bulbificação (duas aplicações) e fim da bulbificação (uma aplicação). As pulverizações iniciaram-se em 10 de outubro de 1997 e finalizaram-se em 31 de outubro de 1997. Como fonte de oxicloreto de Cu utilizou-se o fungicida Recop, Sandoz S.A., e como fonte de ácido bórico utilizou-se o ácido bórico solúvel, $17 \%$ B, ProduQuímica Indústria e Comércio Ltda. As pulverizações foram realizadas com o pulverizador costal Jacto, $20 \mathrm{~L}$. Volume de calda utilizada $500 \mathrm{~L} \mathrm{ha}^{-1}$. No momento da aplicação em campo, utilizou-se um anteparo constituído de papelão comum, para se evitar a deriva de produtos. A colheita ocorreu em 6 de novembro de 1997, uma semana após o término das pulverizações. As plantas foram retiradas do campo e secadas em ambiente semi-sombreado. Após uma semana, as ramas foram cortadas e iniciaram-se as análises. Os bulbos foram armazenados à temperatura ambiente $\left(25^{\circ} \mathrm{C}\right) \mathrm{em}$ delineamento experimental com blocos casualisados, na Estação Experimental de Hortaliças SVS, durante período superior a 90 dias após a colheita. Os bulbos foram acondicionados em caixas plásticas ventiladas.

Delineamento estatístico: $O$ delineamento experimental utilizado foi o de blocos inteiramente casualisados em esquema de parcela subdivididas, sendo as parcelas composta pelos tratamentos e as subparcelas pelos materiais genéticos, utilizando-se 4 repetições, contendo 50 plantas cada. A análise da variância permitiu verificar o efeito dos fatores (tratamento, cultivar e interação) e a comparação entre as médias foi feita pelo teste de Tukey, em nível de significância de 5\%. Em todas as análises, realizou-se o teste de normalidade e homogeneidade das variâncias sobre os dados, para verificação das pressuposições do modelo.

Coleta de dados biométricos: Periodicamente durante o armazenamento, foram acompanhadas as alterações físico-químicas nos bulbos de cebola mediante a medição de alguns parâmetros, a saber: perda de peso, diâmetro, resistência da casca, firmeza e coloração da casca.

Perda de peso: A perda de peso (\%) foi estudada pela relação entre a diferença entre o peso inícial e o final, dividido pelo peso inicial e multiplicada por 100. As pesagens iniciaram após o término da cura, e o peso inicial utilizado referia-se sempre à última medição. As pesagens foram realizadas em balança de precisão, Marte, excluindo-se as perdas em virtude de deterioração e da brotação. Utilizou-se a média de cinco bulbos para cada repetição.

Resistência da casca: A resistência da casca foi determinada no Laboratório Central do Departamento de Planejamento Alimentar e Nutrição da Faculdade de Engenharia de Alimentos, da Universidade Estadual de Campinas, UNICAMP. Para esta determinação utilizou-se texturômetro Stable Micro Systems, Godalming, Surrey, United Kingdon. A medida foi destrutiva e realizada no ponto mediano sobre o equador de cada bulbo. Nesse teste de ruptura, utilizou-se uma sonda de formato pontiagudo, modelo SMS P/2N, com uma força constante de 0,20 N, até a profundidade máxima de penetração de $0,3 \mathrm{~mm}$. 
Cada repetição foi composta de cinco bulbos. A espessura máxima $(0,3 \mathrm{~mm})$ foi baseada na média da espessura da casca externa das variedades e híbridos em estudo.

Firmeza: A análise de firmeza também foi realizada no Laboratório Central do Departamento de Planejamento Alimentar e Nutrição da Faculdade de Engenharia de Alimentos, Universidade Estadual de Campinas, UNICAMP. Para este teste utilizou-se texturômetro Stable Micro Systems, Godalming, Surrey, United Kingdom realizada no ponto mediano sobre o equador de cada bulbo. Utilizou-se uma sonda de formato esférico, modelo P/0-55, com diâmetro de $1 \mathrm{~cm}$, à qual se aplicou uma força constante de $0,20 \mathrm{~N}$, até a profundidade máxima de $10 \mathrm{~mm}$. Cada repetição foi composta pela média das medições realizadas em cinco bulbos.

Cor: A cor externa foi determinada em colorímetro Hunter Lab - Color Quest II ${ }^{\mathrm{TM}}$ II Sphere, Color Quest ${ }^{\mathrm{TM}}$ 45/0, LabScan $^{\mathrm{R}}$, MiniScan ${ }^{\mathrm{TM}}$, Hunter Associates Laboratory, Inc. Reston, Virginia, USA. Esta medida visou avaliar as alterações na cor dos bulbos durante o armazenamento. Utilizou-se para cada repetição a média das medições realizadas em cinco bulbos. As medições foram realizadas no mesmo bulbo, na parte mediana e em faces opostas, durante o período de armazenagem. A escala CIELAB ( $L^{*}$, $\left.a^{*}, b^{*}\right)$ foi utilizada. A cor é relatada por 3 diferentes parâmetros integralizados em um diagrama tridimensional que mostra a coloração do produto. $L^{*}$ varia de 0 a 100 , em que o valor 0 indica o preto e o valor 100 , o branco. Valor $a^{*}$ varia do vermelho $\left(+a^{*}\right)$, localizado a $0^{\circ}$ ou $360^{\circ}$, ao verde $\left(-a^{*}\right)$, que está a $180^{\circ}$ (na ausência dos componentes amarelo ou azul). O valor $b^{*}$, na ausência dos componentes verde ou vermelho, varia do amarelo $\left(+b^{\star}\right)$, e azul $\left(-b^{*}\right)$, que estão a $90^{\circ}$ e $270^{\circ}$ respectivamente. (Shewfelt et al., 1988). Medições de coloração foram expressas em termos de valor (L), ângulo hue $\left(\tan ^{-1} \mathrm{~b}^{*} /\right.$ $a^{*}$ ) e chroma $\left(a^{* 2}+b^{* 2}\right)^{1 / 2}$ (McGuire, 1992, Shewfelt et al., 1988). O parâmetro $L^{*}$ indica a variação da coloração de preta a branca, em uma escala de 0 a 100. Alto valor de $L^{*}$ representa branco a claro (100), baixo valor de $L^{*}$ representa coloração escura a preta (0) (Lancaster et al., 1997; Shewfelt et al., 1988). O ângulo hue, mostra a localização da cor em um diagrama, aonde o ângulo $0^{\circ}$ representa vermelho puro, o $90^{\circ}$ representa o amarelo puro, o de $180^{\circ}$ o verde puro e $0270^{\circ}$ o azul (Shewfelt et al., 1988). Chroma é descrito como a intensidade ou saturação de cor, e é definido pela distância do ângulo hue do centro do diagrama tridimensional. (McGuire, 1992; Shewfelt et al., 1988).

\section{RESULTADOS E DISCUSSÃO}

Perda de peso: Foram realizadas duas avaliações relativas a perda de peso, aos 25 e 45 dias após a colheita. A colheita dos bulbos ocorreu em 6 de novembro e a primeira pesagem dos mesmos ocorreu dez dias após, com a segunda 15 dias após esta, e a terceira 20 dias após esta última. Os resultados apresentados na TABELA 2 mostram que somente na primeira avaliação, 25 dias após a colheita, os bulbos das plantas tratadas com oxicloreto de Cu na dosagem de $250 \mathrm{~g}$ $100 \mathrm{~L}^{-1}$ de água, apresentaram menor perda em peso, que os produzidos pelas plantas que receberam tratamento com

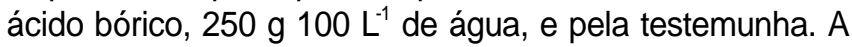
combinação, ácido bórico e oxicloreto de $\mathrm{Cu}$, mostrou maior perda em peso. Na avaliação feita aos 45 dias os tratamentos não mostraram efeito significativo. Não se detectou interação entre o efeito dos tratamentos e os materiais genéticos. $\mathrm{Na}$ primeira avaliação, a menor perda de peso foi observada nos bulbos cujas plantas receberam o tratamento com oxicloreto de cobre, o que pode estar relacionado à maior resistência e espessura da casca. Knott (1933) relaciona a aplicação de Cu com aumentos da espessura e aderência da casca e da firmeza do bulbo. A casca da cebola funciona como uma barreira contra a perda de água. Sem a casca, a perda de peso durante armazenagem pode dobrar (Apeland, 1971). As cultivares, para perda de peso, podem ser divididas por uma escala em que os bulbos das variedades Crioula, Régia e Serrana e o híbrido HS-2 perderam mais peso, e os híbridos HT e HS-1 perderam menos peso, nas duas épocas de avaliação. A maior perda em peso para a variedade Crioula, na primeira avaliação, pode estar relacionada ao ponto de colheita. Todas as cultivares foram colhidas na mesma época e como a variedade Crioula, apesar de apresentar naquele período o estalo das folhas, possui ciclo mais tardio que as demais, e portanto não estava amadurecida fisiológicamente. Esta situação pode ter provocado a maior perda após 25 dias. Por sua vez, o híbrido HS-1 apresentava um excelente e uniforme estalo, o que pode ter contribuído para uma menor perda. Este híbrido é proveniente do cruzamento da variedade Crioula com uma linhagem macho-estéril Baia-Periforme. Para perda de peso, o híbrido diferiu totalmente da variedade polinizadora.

Resistência da casca: Para medir a resistência da casca, as medições foram realizadas no texturômetro, utilizando-se 0 teste de ruptura (força medida em Newtons). A primeira avaliação ocorreu 40 dias após a colheita e a segunda avaliação ocorreu 70 dias após a colheita. Pela TABELA 3 observa-se que para a primeira avaliação, 40 dias após a colheita, bulbos tratados com oxicloreto de $\mathrm{Cu}, 250 \mathrm{~g} 100 \mathrm{~L}^{-}$ ${ }^{1}$ de água, necessitaram maior força para penetração na casca, portanto demonstrando maior resistência, diferindo estatisticamente dos demais. Aplicações com sulfato de $\mathrm{Cu}$ via solo, proporcionam aumento na aderência e espessura da casca (Bender, 1993; Knott, 1933). O tratamento com ácido bórico resultou na segunda maior força para penetração na casca e pela análise estatística valor intermediário aos demais. O B possuí alta influência na formação da casca da cebola (Calbo et al., 1986). A testemunha e o tratamento proveniente da associação entre oxicloreto de $\mathrm{Cu}$ e ácido bórico, demonstraram menor resistência das camadas externas. Esses resultados confirmam o efeito dos tratamentos com micronutrientes para espessura da casca. Em relação as cultivares utilizadas, a variedade Crioula, 
TABELA 2 - Perda em peso (\%), em seis cultivares de cebola, submetidos a tratamentos pré-colheita em experimento instalado na Estação Experimental de Hortaliças SVS, Paulínia, Estado de São Paulo.

\begin{tabular}{|c|c|c|c|c|c|}
\hline \multicolumn{6}{|c|}{ PRIMEIRA AVALIAÇÃO (25 dias após a colheita) } \\
\hline CULTINAR & Testemunha ${ }^{\mathrm{L} 1}$ & $\begin{array}{l}\text { Oxicloreto de } \mathrm{Cu} \\
250{\mathrm{~g} 100 \mathrm{~L}^{-1}}\end{array}$ & $\begin{array}{l}\text { Ácido bórico } \\
250{\mathrm{~g} 100 \mathrm{~L}^{-1}}^{2}\end{array}$ & $\begin{array}{l}\text { Ox. de Cu 250g } 100 \mathrm{~L}^{-1}+ \\
\text { ác. bórico } 250 \mathrm{~g} 100 \mathrm{~L}^{-1}\end{array}$ & $\begin{array}{c}\text { Média } \\
\text { DMS } 0,41\end{array}$ \\
\hline Serrana & $2,25^{\llcorner 2}$ & 1,66 & 3,36 & 2,95 & $2,51 a b$ \\
\hline Régia & 3,23 & 3,38 & 1,69 & 2,82 & $2,74 a b$ \\
\hline Crioula & 2,58 & 2,93 & 3,26 & 3,49 & $3,05 \mathrm{a}$ \\
\hline HT & 2,00 & 1,37 & 2,29 & 1,54 & $1,78 \mathrm{bc}$ \\
\hline HS-1 & 1,47 & 1,04 & 1,71 & 1,18 & $1,34 \mathrm{c}$ \\
\hline HS-2 & 2,98 & 2,34 & 2,07 & 3,41 & $2,68 a b$ \\
\hline Média DMS 0,14 & $2,38 \mathrm{AB}$ & $2,04 \mathrm{~B}$ & $2,35 \mathrm{AB}$ & $2,48 \mathrm{~A}$ & 2,31 \\
\hline CVA $4,2 \%$ & & & & & CVB $25,82 \%$ \\
\hline \multicolumn{6}{|c|}{ SEGUNDA AVALIAÇÃO (45 dias após a colheita) } \\
\hline CULTINAR & Testemunha $^{\mathrm{L} 1}$ & $\begin{array}{c}\text { Oxicloreto de } \\
\text { Cu } 250 \mathrm{~g} 100 \mathrm{~L}^{-1}\end{array}$ & $\begin{array}{l}\text { Ácido bórico } \\
250{\mathrm{~g} 100 \mathrm{~L}^{-1}}^{-1}\end{array}$ & 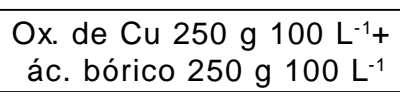 & $\begin{array}{c}\text { Média } \\
\text { DMS } 0,45\end{array}$ \\
\hline Serrana & $5,72^{\mathrm{L} 2}$ & 4,49 & 5,57 & 5,27 & $5,25 a b$ \\
\hline Régia & 5,13 & 5,31 & 5,73 & 10,19 & $6,50 \mathrm{a}$ \\
\hline Crioula & 5,39 & 5,90 & 4,64 & 4,00 & $4,96 a b$ \\
\hline $\mathrm{HT}$ & 3,79 & 4,14 & 4,14 & 3,74 & $3,95 \mathrm{bc}$ \\
\hline HS-1 & 3,10 & 2,94 & 2,36 & 4,28 & $3,13 \mathrm{c}$ \\
\hline HS-2 & 3,35 & 3,62 & 3,19 & 5,30 & $3,83 \mathrm{bc}$ \\
\hline Média DMS 0,37 & $4,38 \mathrm{~A}$ & $4,34 \mathrm{~A}$ & $4,18 \mathrm{~A}$ & $5,29 \mathrm{~A}$ & 4,55 \\
\hline CVA $8,25 \%$ & & & & & CVB $20,25 \%$ \\
\hline
\end{tabular}

${ }^{\mathrm{L}}{ }_{\mathrm{1}}^{\mathrm{m}} \mathrm{d}$ dias seguidas por letras distintas maiúsculas na linha e minúsculas na coluna, diferem a $5 \%$ pelo teste de Tukey.

${ }^{\text {L2} d a d o s ~ f o r a m ~ t r a n s f o r m a d o s ~ p a r a ~ a v a l i a c ̧ a ̃ o ~}$

CVA - coeficiente de variação da parcela

CVB - coeficiente de variação da subparcela

sabidamente conhecida pela sua película externa, demonstrou a maior resistência a penetração, seguida pelos híbridos HS-1 e HT. A variedade Régia demonstrou a menor resistência à penetração, o que deve estar relacionada à baixa conservação do material e, portanto ao fácil desprendimento das películas externas. Doruchowski (1986) relata que a espessura de casca mostrou baixo coeficiente de herdabilidade e alta variabilidade entre as linhagens para essa característica. Observou-se alta influência do ambiente para essas características. Outro parâmetro relacionado à casca, estudado por esse autor, foi aderência da casca. Para esse parâmetro sugere-se uma dominância parcial dos materiais com casca mais aderente nos híbridos $F_{1}$. Linhagens de alta variabilidade para essa característica demonstraram nos híbridos $F_{1}$, alta variabilidade e alta influência às mudanças ambientais. Para obtenção de híbridos com casca bem aderida, o autor sugere que ambos os parentais possuam essa característica. Nesta avaliação observou-se que dois híbridos, no qual a variedade Crioula foi utilizada como polinizadora, apresentaram maior resistência da casca. Para a segunda avaliação (TABELA 3), os tratamentos utilizados não demonstraram efeitos significativos para aumento da resistência da camada externa após um período prolongado de armazenamento, superior a 70 dias. A média da força utilizada na segunda medição para ruptura da casca foi cerca de $13 \%$ menor do que na primeira medição. Essa perda de resistência e aderência das películas externas nas condições tropicais durante 0 armazenamento é descrita e observada por produtores. A variedade Crioula continuou apresentando maior resistência da camada externa. Os híbridos HS-1, HS-2 e HT e a variedade Régia apresentaram valores intermediários. A variedade Serrana apresentou a menor resistência da camada externa.

Firmeza: Para se medir a firmeza dos bulbos submetidos aos diferentes tratamentos, as análises foram realizadas com a seguinte periodicidade: 25 e 60 dias após a colheita. As medições foram realizadas no texturômetro, através do teste de compressão e a força medida em Newtons. Nas duas avaliações citadas não se observou resposta para os tratamentos utilizados (TABELA 4). Os resultados encontrados conflitaram com os observados por Knott 
TABELA 3 - Espessura de casca (Força expressa em Newtons) em 6 cultivares de cebola submetidos a tratamentos précolheita em experimento instalado na Estação Experimental de Hortaliças SVS, Paulínia, Estado de São Paulo.

\begin{tabular}{|c|c|c|c|c|c|}
\hline \multicolumn{6}{|c|}{ PRIMEIRA AVALIAÇÃO (40 dias após a colheita) } \\
\hline CULTIVAR & Testemunha & $\begin{array}{l}\text { Oxicloreto de } \mathrm{Cu} \\
250{\mathrm{~g} 100 \mathrm{~L}^{-1}}^{-1}\end{array}$ & $\begin{array}{l}\text { Ácido bórico } \\
250{\mathrm{~g} 100 \mathrm{~L}^{-1}}^{2}\end{array}$ & $\begin{array}{l}\text { Ox. de Cu } 250 \mathrm{~g} 100 \mathrm{~L}^{-1}+ \\
\text { ác. bórico } 250 \mathrm{~g} 100 \mathrm{~L}^{-1}\end{array}$ & $\begin{array}{c}\text { Média } \\
\text { DMS } 0,35\end{array}$ \\
\hline Serrana & $1,76^{*}$ & 1,89 & 1,88 & 1,83 & $1,84 \mathrm{bc}$ \\
\hline Régia & 1,51 & 1,96 & 1,50 & 1,91 & $1,72 \mathrm{c}$ \\
\hline Crioula & 2,30 & 2,58 & 2,34 & 2,01 & $2,31 \mathrm{a}$ \\
\hline HT & 1,80 & 2,25 & 2,07 & 1,75 & $1,97 \mathrm{abc}$ \\
\hline HS-1 & 2,01 & 2,22 & 2,16 & 2,25 & $2,16 a b$ \\
\hline HS-2 & 2,03 & 1,86 & 1,94 & 1,63 & $1,86 \mathrm{bc}$ \\
\hline Média DMS 0,22 & $1,90 \mathrm{~B}$ & $2,13 \mathrm{~A}$ & $1,98 \mathrm{AB}$ & $1,90 \mathrm{~B}$ & 1,98 \\
\hline CVA $5,01 \%$ & & & & & CVB $17,29 \%$ \\
\hline
\end{tabular}

SEGUNDA AVALIAÇÃO (70 dias após a colheita)

\begin{tabular}{|c|c|c|c|c|c|}
\hline CULTIVAR & Testemunha & $\begin{array}{l}\text { Oxicloreto de } \mathrm{Cu} \\
250 \mathrm{~g} 100 \mathrm{~L}^{-1}\end{array}$ & $\begin{array}{l}\text { Ácido bórico } \\
250 \mathrm{~g} 100 \mathrm{~L}^{-1}\end{array}$ & $\begin{array}{l}\text { Ox. de Cu } 250 \mathrm{~g} 100 \mathrm{~L}^{-1}+ \\
\text { ác. bórico } 250 \mathrm{~g} 100 \mathrm{~L}^{-1}\end{array}$ & $\begin{array}{c}\text { Média } \\
\text { DMS } 0,36\end{array}$ \\
\hline Serrana & $1,55^{*}$ & 1,44 & 1,55 & 1,55 & $1,52 \mathrm{~b}$ \\
\hline Régia & 1,69 & 1,51 & 1,81 & 1,27 & $1,57 \mathrm{ab}$ \\
\hline Crioula & 1,83 & 2,14 & 2,08 & 1,66 & $1,93 \mathrm{a}$ \\
\hline HT & 1,31 & 1,88 & 2,02 & 1,65 & $1,72 a b$ \\
\hline HS-1 & 1,84 & 1,81 & 1,64 & 2,07 & $1,84 \mathrm{ab}$ \\
\hline HS-2 & 1,82 & 1,75 & 1,72 & 1,64 & $1,73 a b$ \\
\hline Média DMS 0,18 & $1,68 \mathrm{~A}$ & $1,75 \mathrm{~A}$ & $1,80 \mathrm{~A}$ & $1,64 \mathrm{~A}$ & 1,72 \\
\hline CVA $4,67 \%$ & & & & & CVB $20,14 \%$ \\
\hline
\end{tabular}

*médias seguidas por letras distintas maiúsculas na linha e minúsculas na coluna, diferem a $5 \%$ pelo teste de Tukey.

CVA - coeficiente de variação da parcela - tratamentos

CVB - coeficiente de variação da subparcela - cultivares

(1933) em que aplicações com sulfato de Cu aumentaram a firmeza dos bulbos. As cultivares testadas apresentaram diferentes respostas para o teste de compressão, demonstrando uma variação quanto à firmeza dos bulbos. Na primeira avaliação, 25 dias após a colheita, observouse maior firmeza para os bulbos da variedade Crioula (TABELA 4). Na segunda avaliação, 60 dias após a colheita, a variedade Crioula continuou apresentando bulbos mais firmes que as demais variedades, mas não diferindo estatisticamente dos híbridos. Doruchowski (1986) descreve analisando populações $F_{1}$, que menor herdabilidade foi demonstrada para o parâmetro firmeza (32-48\%). Por sua vez, observa-se que os híbridos provenientes do cruzamento utilizando-se a variedade Crioula como polinizadora apresentaram bulbos firmes, quando comparado às variedades Serrana e Régia.

Cor: Para a medição da coloração dos bulbos foram realizadas três avaliações, aos 7, 40 e 60 dias após a colheita. A cor dos bulbos está expressa através dos parâmetros L, ângulo hue e chroma. Para a primeira avaliação, 7 dias após a colheita, e a segunda avaliação
40 dias após a colheita, não ocorreram diferenças significativas para os parâmetros $L$, ângulo hue e chroma nos tratamentos utilizados (dados não apresentados). Bulbos armazenados demonstraram aumento no teor de qüercitina durante o armazenamento (Patil et al., 1995). Portanto a não resposta aos tratamentos utilizados pode estar relacionada ao pouco tempo de armazenamento. Observa-se pela TABELA 5, terceira avaliação, 60 dias após a colheita, que não houve diferença significativa entre os tratamentos utilizados, para o valor $\mathrm{L}$. Em relação às cultivares, novamente se repetiu o encontrado nas duas avaliações anteriores. A variedade Crioula apresentou menor valor para $L$, representando uma coloração mais escura. A variedade Serrana apresentou maior valor para $\mathrm{L}$, apresentando coloração mais clara. Os híbridos e a variedade Régia apresentaram valores intermediários para coloração de casca. Para o parâmetro ângulo hue (TABELA 5) ocorreu uma interação entre o efeito dos tratamentos químicos e o material genético. Observando as diferenças entre os tratamentos, nota-se que a variedade Régia apresentou menor valor para o ângulo

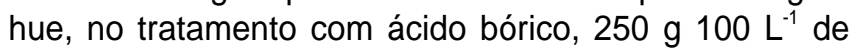


TABELA 4 - Firmeza dos bulbos (Força expressa em Newtons) em 6 cultivares de cebola submetidos a tratamentos précolheita no em experimento instalado na Estação Experimental de Hortaliças SVS, Paulínia, Estado de São Paulo.

\begin{tabular}{lccccc}
\hline \multicolumn{7}{c}{ SEGUNDA AVALIAÇÃO (25 dias após a colheita) } \\
\hline CULTIVAR & Testemunha & $\begin{array}{c}\text { Oxicloreto de Cu } \\
250 \mathrm{~g} 100 \mathrm{~L}^{-1}\end{array}$ & $\begin{array}{c}\text { Ácido bórico } \\
250 \mathrm{~g} 100 \mathrm{~L}^{-1}\end{array}$ & $\begin{array}{c}\text { Ox. de Cu } 250 \mathrm{~g} 100 \mathrm{~L}^{-1}+ \\
\text { ác. bórico } 250 \mathrm{~g} 100 \mathrm{~L}^{-1}\end{array}$ & $\begin{array}{c}\text { Média } \\
\text { DMS } 0,36\end{array}$ \\
\hline Serrana & $1,55^{*}$ & 1,44 & 1,55 & 1,55 & $1,52 \mathrm{~b}$ \\
Régia & 1,69 & 1,51 & 1,81 & 1,27 & $1,57 \mathrm{ab}$ \\
Crioula & 1,83 & 2,14 & 2,08 & 1,66 & $1,93 \mathrm{a}$ \\
HT & 1,31 & 1,88 & 2,02 & 1,65 & $1,72 \mathrm{ab}$ \\
HS-1 & 1,84 & 1,81 & 1,64 & 2,07 & $1,84 \mathrm{ab}$ \\
HS-2 & 1,82 & 1,75 & 1,72 & 1,64 & $1,73 \mathrm{ab}$ \\
Média DMS 0,18 & $1,68 \mathrm{~A}$ & $1,75 \mathrm{~A}$ & $1,80 \mathrm{~A}$ & $1,64 \mathrm{~A}$ & 1,72 \\
\hline CVA 4,67\% & \multicolumn{7}{c}{} & & CVB 20,14\%
\end{tabular}

SEGUNDA AVALIAÇÃO (60 dias após a colheita)

\begin{tabular}{|c|c|c|c|c|c|}
\hline CULTIVAR & Testemunha & $\begin{array}{l}\text { Oxicloreto de } \mathrm{Cu} \\
250{\mathrm{~g} 100 \mathrm{~L}^{-1}}^{-1}\end{array}$ & $\begin{array}{l}\text { Ácido bórico } \\
250{\mathrm{~g} 100 \mathrm{~L}^{-1}}^{-1}\end{array}$ & $\begin{array}{l}\text { Ox. de Cu } 250 \mathrm{~g} 100 \mathrm{~L}^{-1}+ \\
\text { ác. bórico } 250 \mathrm{~g} 100 \mathrm{~L}^{-1}\end{array}$ & $\begin{array}{l}\text { Média } \\
\text { DMS } \\
12,50^{* * *}\end{array}$ \\
\hline Serrana & $123,66^{*}$ & 136,43 & 125,35 & 118,81 & $126,06 \mathrm{~b}$ \\
\hline Régia & 107,77 & 109,74 & 111,24 & 110,73 & $109,87 \mathrm{c}$ \\
\hline Crioula & 144,90 & 153,15 & 162,53 & 161,85 & $155,61 \mathrm{a}$ \\
\hline HT & 154,22 & 141,94 & 147,39 & 139,70 & $145,81 \mathrm{a}$ \\
\hline HS-1 & 151,87 & 145,16 & 155,19 & 157,06 & $152,32 \mathrm{a}$ \\
\hline HS-2 & 136,87 & 151,50 & 152,65 & 155,12 & $149,03 \mathrm{a}$ \\
\hline Média DMS 9,89** & $136,55 \mathrm{~A}$ & $139,65 \mathrm{~A}$ & $142,39 \mathrm{~A}$ & $140,55 \mathrm{~A}$ & 139,78 \\
\hline CVA $3,2 \%$ & & & & & CVB $8,6 \%$ \\
\hline
\end{tabular}

*médias seguidas por letras distintas maiúsculas na linha e minúsculas na coluna, diferem a $5 \%$ pelo teste de Tukey.

CVA - coeficiente de variação da parcela- tratamentos

CVB - coeficiente de variação da subparcela- cultivares

água, significativamente diferente dos outros dois tratamentos: combinação oxicloreto de Cu e ácido bórico e oxicloreto de $\mathrm{Cu}, 250 \mathrm{~g} 100 \mathrm{~L}^{-1}$ de água. Valores menores para o ângulo hue indicam coloração mais próxima do avermelhado. A testemunha apresentou maiores valores do ângulo hue, indicando coloração mais próxima do amarelo. Portanto, observa-se efeito dos tratamentos com micronutrientes na mudança de coloração da cebola nesta avaliação. Tanto Cu (Knott, 1933; Nelson et al., 1956; Murphy \& Walsh, 1973), quanto B (Calbo et al., 1986) influenciam a coloração da casca de cebola. A variedade Régia apresentou nas duas avaliações anteriores, para o parâmetro hue, valores indicativos para coloração mais próximo ao amarelo. Nesta avaliação, os valores apresentados estão mais próximos ao vermelho que a testemunha, representando efeito do tratamento. Além da influência dos tratamentos, o período de armazenamento (Patil et al., 1995) e a temperatura (Cardoso et al., 1995) podem ter influenciado a alteração da cor. Analisando separadamente cada tratamento, observa-se que no

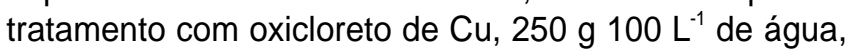
a variedade Crioula apresentou o menor valor do ângulo hue, estatisticamente diferente dos demais, indicando portanto, maior aproximação com a coloração vermelha. Observa-se comportamento semelhante no tratamento combinado, oxicloreto de cobre e ácido bórico. $\mathrm{Na}$ testemunha e no tratamento com ácido bórico, a variedade Crioula e os híbridos demonstraram valores semelhantes, não diferindo estatisticamente. Observa-se para a variedade Serrana maior valor para hue em todos os tratamentos, portanto, maior aproximação com a cor amarela. Para o parâmetro chroma (TABELA 6), o

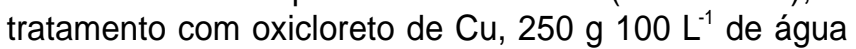
demonstrou maiores valores, significativamente diferentes dos demais tratamentos, independentemente da cultivar utilizada. Bender (1993) relata que a deficiência em Cu pode proporcionar bulbos de coloração mais clara. Maiores valores de chroma indicam maior intensidade luminosa. Os

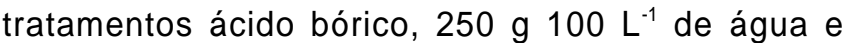
associação oxicloreto de Cu e ácido bórico demonstraram valores intermediários, superiores à testemunha. Entre as cultivares utilizadas o maior valor de chroma foi apresentado no híbrido HS-1, seguido pela variedade Crioula e pelos híbridos HS-2 e HT. A variedade Régia 
TABELA 5 - Resultados da terceira avaliação para coloração,parâmetro L e ângulo Hue realizada aos 60 dias após a colheita em 6 cultivares de cebola submetidos a tratamentos pré-colheita em experimento instalado na Estação Experimental de Hortaliças SVS, Paulínia, estado de São Paulo.

\begin{tabular}{|c|c|c|c|c|c|}
\hline \multicolumn{6}{|c|}{$\mathrm{L}$} \\
\hline CULTIVAR & Testemunha & $\begin{array}{l}\text { Oxicloreto de Cu } \\
250{\mathrm{~g} 100 \mathrm{~L}^{-1}}^{-1}\end{array}$ & $\begin{array}{l}\text { Ácido bórico } \\
250{\mathrm{~g} 100 \mathrm{~L}^{-1}}^{-1}\end{array}$ & $\begin{array}{l}\text { Ox. de Cu } 250 \mathrm{~g} 100 \mathrm{~L}^{-1}+ \\
\text { ác. bórico } 250 \mathrm{~g} 100 \mathrm{~L}^{-1}\end{array}$ & $\begin{array}{c}\text { Média } \\
\text { DMS 3,07 }\end{array}$ \\
\hline Serrana & $60,44^{*}$ & 60,51 & 59,00 & 58,60 & 59,64 a \\
\hline Régia & 59,39 & 55,61 & 54,93 & 59,21 & $57,28 \mathrm{ab}$ \\
\hline Crioula & 52,02 & 52,36 & 52,11 & 52,63 & $52,28 \mathrm{c}$ \\
\hline HT & 56,93 & 57,77 & 58,63 & 59,22 & $58,13 a b$ \\
\hline HS-1 & 55,82 & 58,71 & 58,64 & 54,86 & $57,00 a b$ \\
\hline HS-2 & 53,02 & 55,77 & 58,82 & 57,65 & $56,31 \mathrm{~b}$ \\
\hline Média DMS 3,28 & $56,27 \mathrm{~A}$ & $56,79 \mathrm{~A}$ & $57,02 \mathrm{~A}$ & $57,03 \mathrm{~A}$ & 56,77 \\
\hline CVA $2,61 \%$ & & & & & CVB $5,2 \%$ \\
\hline \multicolumn{6}{|c|}{ ÂNGULO HUE } \\
\hline CULTIVAR & Testemunha & $\begin{array}{l}\text { Oxicloreto de } \mathrm{Cu} \\
250{\mathrm{~g} 100 \mathrm{~L}^{-1}}^{-1}\end{array}$ & $\begin{array}{l}\text { Ácido bórico } 250 \\
\text { g } 100 \mathrm{~L}^{-1}\end{array}$ & $\begin{array}{l}\text { Ox. de Cu } 250 \mathrm{~g} 100 \mathrm{~L}^{-1}+ \\
\text { ác. bórico } 250{\mathrm{~g} 100 \mathrm{~L}^{-1}}\end{array}$ & $\begin{array}{c}\text { Média DMS } \\
11,39\end{array}$ \\
\hline Serrana & $70,89 a A^{* * * *}$ & 67,66 aA & $71,38 \mathrm{aA}$ & $64,17 \mathrm{aA}$ & 68,52 \\
\hline Régia & 66,96 aA & 62,79 abAB & $56,75 \mathrm{bB}$ & $59,96 \mathrm{abAB}$ & 61,61 \\
\hline Crioula & $49,23 \mathrm{bA}$ & $48,92 \mathrm{cA}$ & $48,82 \mathrm{bA}$ & $50,05 \mathrm{bA}$ & 49,25 \\
\hline HT & $54,13 \mathrm{bA}$ & $60,39 a b A$ & $59,01 \mathrm{bA}$ & $58,21 a b A$ & 57,94 \\
\hline HS-1 & $52,08 \mathrm{bA}$ & $60,56 \mathrm{abA}$ & $54,40 \mathrm{bA}$ & $51,35 \mathrm{bA}$ & 54,60 \\
\hline HS-2 & $56,01 \mathrm{bA}$ & $52,80 \mathrm{bcA}$ & $56,79 \mathrm{bA}$ & $59,17 \mathrm{abA}$ & 56,19 \\
\hline Média DMS 10,53 & 58,22 & 58,85 & 57,86 & 57,15 & 58,02 \\
\hline CVA 3,93\% & & & & & CVB $8,73 \%$ \\
\hline
\end{tabular}

*médias seguidas por letras distintas maiúsculas na linha e minúsculas na coluna, diferem a $5 \%$ pelo teste de Tukey.

**** médias seguidas por letras distintas maiúsculas e minúsculas na linha diferem a $5 \%$ pelo teste de Tukey.

CVA - coeficiente de variação da parcela - tratamentos

CVB - coeficiente de variação da subparcela - cultivares

apresentou valor intermediário entre os híbridos (HS-2 e HT) e a variedade Serrana, que apresentou o menor valor. A maior resposta aos tratamentos nesta terceira avaliação para os parâmetros ângulo hue e chroma pode estar relacionada ao fato que durante o armazenamento ocorre o aumento da qüercitina, principal pigmento responsável pela coloração dos bulbos de cebola (Patil et al., 1995), o que pode explicar valores mais significativos após 2 meses de armazenagem.

\section{CONCLUSÕES}

Os tratamentos pré-colheita, com os micronutrientes $\mathrm{Cu}$ e $\mathrm{B}$ aplicados durante a bulbificação da cebola, produziram melhoria na qualidade dos bulbos colhidos no experimento em 1997. Bulbos tratados com micronutrientes apresentaram incremento na coloração e aumento da resistência da casca e menor perda de peso durante $o$ armazenamento. As seguintes conclusões foram observadas: (a) A aplicação em pré-colheita de oxicloreto

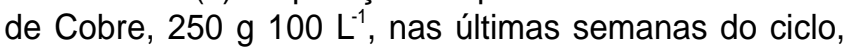
aumentou significativamente a resistência da casca do bulbo para todos as cultivares; (b) 0 tratamento pré-

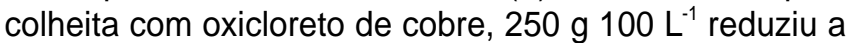

perda de peso dos bulbos para todas as cultivares; (c) Os tratamentos pré-colheita com oxicloreto de cobre, $250 \mathrm{~g}$ $100 \mathrm{~L}^{-1}$ incrementaram a intensidade da cor e acentuaram a coloração avermelhada em todas as cultivares; (d) Os

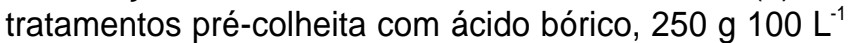
acentuaram a coloração avermelhada da variedade Régia; (e) Cultivares de casca mais escura obtiveram melhor resposta aos tratamentos pré-colheita para coloração do que cultivares de cor mais clara; (f) Para o parâmetro firmeza, não foi observada nenhuma influência dos tratamentos pré-colheita; $(g)$ Híbridos $F_{1}$ provenientes de cruzamento, cujo polinizador possuía casca de coloração escura e espessa, demonstraram valores intermediários para coloração e espessura.

\section{REFERÊNCIAS BIBLIOGRÁFICAS}

AcostA, A.; GAVIOLA, J.C.; GALMARINI, C. Producción de semilla de cebolla. La Consulta: Asociación Cooperadora EEA La Consulta, 1993. 83p.

APELAND, J. Effects of scale quality in physiological processes in onion. Acta Horticulturae, v. 20, p.72-79, 1971.

BENDER, D.A. Vegetable crops-Onion. In: BENNET, W.F (Ed.) Nutrient deficiencies \& toxicities in crop plants. St. Paul: The American Phytopathological Society, 1993. p.131-135. 
TABELA 6 - Resultados da terceira avaliação para coloração, parâmetro Chroma, realizada aos 60 dias após a colheita em 6 cultivares de cebola submetidos a tratamentos pré-colheita em experimento instalado na Estação Experimental de Hortaliças SVS, Paulínia, estado de São Paulo.

\begin{tabular}{|c|c|c|c|c|c|}
\hline \multicolumn{6}{|c|}{ CHROMA } \\
\hline CULTIVAR & Testemunha & $\begin{array}{l}\text { Oxicloreto de } \mathrm{Cu} \\
250 \mathrm{~g} 100 \mathrm{~L}^{-1}\end{array}$ & $\begin{array}{l}\text { Ácido bórico } \\
250{\mathrm{~g} 100 \mathrm{~L}^{-1}}^{2}\end{array}$ & 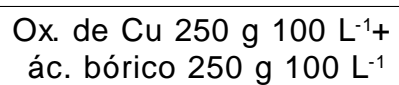 & $\begin{array}{c}\text { Média } \\
\text { DMS } 1,46\end{array}$ \\
\hline Serrana & $21,24^{*}$ & 21,96 & 20,96 & 21,65 & $21,46 \mathrm{c}$ \\
\hline Régia & 22,12 & 22,93 & 21,77 & 23,34 & $22,54 \mathrm{bc}$ \\
\hline Crioula & 23,23 & 24,62 & 22,97 & 23,69 & $23,63 a b$ \\
\hline HT & 23,67 & 22,22 & 23,59 & 23,44 & $23,23 \mathrm{~b}$ \\
\hline HS-1 & 23,83 & 27,12 & 24,44 & 24,07 & 24,86 a \\
\hline HS-2 & 22,64 & 23,30 & 24,15 & 23,28 & $23,34 \mathrm{~b}$ \\
\hline Média DMS 0,73 & $22,79 \mathrm{~B}$ & $23,69 \mathrm{~A}$ & $22,98 \mathrm{AB}$ & $23,24 \mathrm{AB}$ & 23,18 \\
\hline$\overline{C V A} 1,68 \%$ & & & & & CVB $3,60 \%$ \\
\hline
\end{tabular}

*médias seguidas por letras distintas maiúsculas na linha e minúsculas na coluna, diferem a $5 \%$ pelo teste de Tukey.

CVA - coeficiente de variação da parcela

CVB - coeficiente de variação da subparcela

BOEING, G. Cebola. Florianópolis: Instituto de Planejamento e Economia Agrícola de Santa Catarina, 1995. 85p.

BREWSTER, J.L.; BUTLER, H.A. Effects of nitrogen supply on bulb development in onion( Allium cepa, L.). Journal of Experimental Botany, v.40, p.1155-1162, 1989.

CALBO, M.E.R.; MONNERAT, P.H.; SHIMOYA, C. Caracterização de sintomas de deficiência de boro em cebola (Allium cepa L.) em estádio de produção. Revista Ceres, v.33, p.274-280, 1986.

CARDOSO, A.I.I.; DELLA VECCHIA, P.T.; FARIA, L.P. Herança de coloração de bulbos em cebola (Allium cepa L.) com resistência a Colletotrichum gloeosporioides. Scientia Agricola, v.52, p.384-386, 1995.

CROZIER, A.; LEAN, M.E.J.; McDONALD, M.S.; BLACK, C. Quantitative analysis of the flavonoid content of commercial tomatoes, onions, lettuce, and celery. Journal of Agriculture and Food Chemistry, v.45, p.590-595, 1997.

DORUCHOWSKI, R.W. Variability and hereditability of some onion (Allium cepa L.) characteristics of parental forms, $F_{1}$ hybrids and $F_{2}$ generation. Acta Agrobotanica, v.39, p.293-324, 1986.

ELLERBROCK, L.A. Copper requirements for onions grown on organic soils in New York. Acta Horticulturae, n.433, p.567-571, 1997.

FERREIRA, M.D. Cultura da cebola: recomendações técnicas. Asgrow Seed Company, 2000. 25p.

FENWICK, G.R.; HANLEY, A.B. The Genus Allium: Part 2. CRC Critical Reviews In Food Science and Nutrition, v.22, n.4, p.273-377, 1985.

FNP Consultoria \& Comércio. Agrianual 1999. São Paulo, 1999. $521 p$.

FOSSEN, T.; ANDERSEN, A.T.; OVSTEDAL, D.O.; PEDERSEN, A.T.; RAKNES, A. Characteristic anthocyanin pattern from onions and other Allium spp. Journal of Food Science, v.61, p.703-706, 1996.

KNOTT, J.E. The effect of certain mineral elements on the color and thickness of onion scales. Ithaca: Cornell Agricultural Experiment Station, 1933. 14p. (Bulletin 552).
LANCASTER, J.E.; LISTER, C.E.; READY, P.F.; TRIGGS, C.M Influence of pigment composition on skin color in a wide range of fruit and vegetables. Journal of American Society of Horticultural Science, v.122, p.594-598, 1997.

LEIGHTON, T.; GINTHER, C.; FLUSS, L.; HARTER, W.K.; CANSADO, J.; NOTARIO, V. Molecular characterization of quercitin and quercitin glycosides in Allium vegetables. American Chemical Society: Symposium Series, n.507, p.220-238, 1992.

McGUIRE, R.G. Reporting of objective color measurements. HortScience, v.27, p.1254-1255, 1992.

MURPHY, L.S.; WALSH, L.M. Correction of micronutrient deficiencies with fertilizers. In: DINAUER, R.C. (Ed.) Madison: SSSA, 1973. cap.15, p.347-388: Micronutrients in agriculture.

NELSON, L.G.; BERGER, K.C.; ANDRIES, H.J. Copper requirements and deficiency symptoms of a number of field and vegetable crops. Soil Science Society of America Proceedings, v.20, p.69-72, 1956.

PATIL, B.S.; PIKE, L.M.; YOO, K.S. Variation in the quercitin content in different colored onions (Allium cepa L.). Journal of American Society for Horticultural Science, v.120, p.909-913, 1995.

RAIJ, B. van.; CANTARELLA, H.; QUAGGIO, J.A.; FURLANI, A.M.C. Recomendações de adubação e calagem para o Estado de São Paulo. 2.ed. Campinas: IAC, 1996. 285p.

SHEWFELT, R.L.; THAI, C.N.; DAVIS, J.W.. Prediction of changes in color of tomatoes during ripening at different constant temperatures. Journal of Food Science, v.53, p.1433-1437, 1988.

TUCKER, W.G.; DREW, R.L.K. Post-harvest studies on autummdrilled bulb onions. The effect of harvest date, conditioning treatments and field drying on skin quality and on storage performance. Journal of Horticultural Science, v.57, p.339-348, 1982.

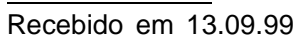

